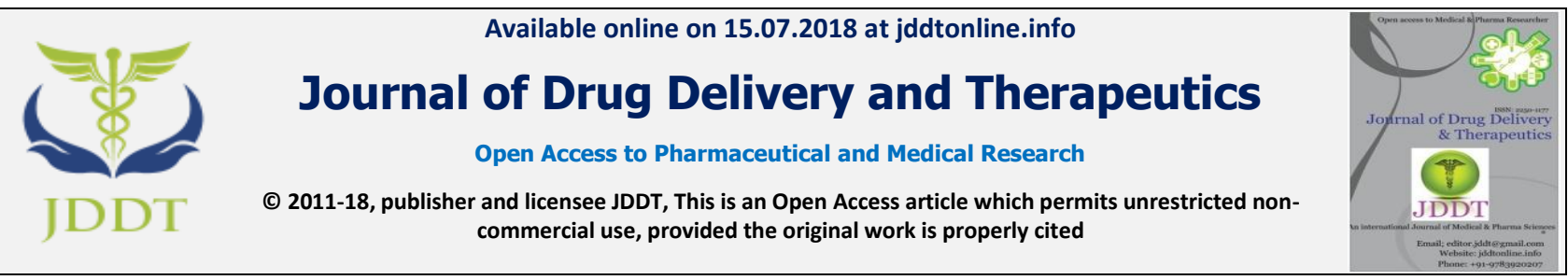

Open $\mathcal{O}_{\text {Access }}$

Review Article

\title{
SOFT CHEWABLE DRUG DELIVERY SYSTEM: ORAL MEDICATED JELLY AND SOFT CHEW
}

\author{
Taranum Ruheena ${ }^{1}$, Mittapally Sirisha ${ }^{2}$ \\ ${ }^{1}$ Student, Department of Pharmaceutics, Deccan School of Pharmacy, Darussalam, Aghapura, Hyderabad-01, Telangana, India \\ ${ }^{2}$ Associate Professor, Department of Pharmaceutics, Deccan School of Pharmacy, Darussalam, Aghapura, Hyderabad-01, \\ Telangana, India
}

\begin{abstract}
Oral route is most commonly used for administration of medicament since they are economical, easy to administer, \& high patient acceptance. Due to difficulty in administration of therapeutic agents to dysphagia patients it becomes necessary to develop a formulation in order to overcome such inconvenience. Jellies are semisolid dosage form they are transparent, non greasy, \& can be used internally as well as externally. Its matrix include large amount of water \& they resemble to mucilage but possess jelly like consistency. Advantages of chewable formulation are that it does not require water for ingestion, aesthetic appearance, and good texture. Soft chew is solid, single dose formulation. It is used as acute medication since it is mobile drug delivery systems. They are formulated using heating \& congealing technique. It serves as novel dosage form with wide applications in pharmaceuticals, nutraceuticals and over the counter medicines
\end{abstract}

Keywords: Jellies, Soft chew, Nutraceuticals

Article Info: Received 11 May, 2018; Review Completed 04 July 2018; Accepted 05 July 2018; Available online 15 July 2018

\section{Cite this article as:}

Taranum R, Mittapally S, Soft chewable drug delivery system: oral medicated jelly and soft chew, Journal of Drug Delivery and Therapeutics. 2018; 8(4):65-72 DOI: http://dx.doi.org/10.22270/jddt.v8i4.1784

\section{${ }^{*}$ Address for Correspondence:}

Taranum Ruheena, Student, Department of Pharmaceutics, Deccan School of Pharmacy, Darussalam, Aghapura Hyderabad-01, Telangana, India.

\section{INTRODUCTION}

\section{Oral Drug Delivery}

In spite of incredible progress in drug delivery, oral route remains the most chosen route for the administration of medicinal agents, since they comprise of low cost of treatment, and effortlessness in administration of therapeutic agent, patient compliance. Moreover it is regarded as most cost-effective and safest technique of drug delivery.

Patients are generally comfortable to orally delivered drugs as this method is non-invasive compared to that of parental delivery of medication. Approximately $80 \%$ of all therapeutic / medicinal agents utilize the oral route. Among various dosage form tablets, capsules and granules persist to remain the dosage form of first preference. Hence it is essential that there should be advancement in oral drug delivery technology in order to meet needs of people \& to deliver safe \& effective treatment to patients. Oral dosage forms represents the majority of the drug-delivery market because of its various advantages such as safety, efficacy, costeffective, and patient compliance in comparison to other routes of delivery. Amongst various pharmaceutical dosage forms, oral dosage forms possess maximum characteristic of ideal dosage forms.

\section{Soft Chewable Drug Delivery}

For the oral administration of drug there are several preparations like tablets, pills, capsules, syrup, etc. Such dosage forms are not easily taken by paediatric and sometimes geriatric patients, particularly patient with dsyphagia. Among these, liquid preparations are easily taken by them compared with other dosage forms. 
However, because of liquid, there are problems to be overcome, when formulating into dosage forms, such as masking of bitterness of effective components, there dispersibility and stability. Paediatrics cannot easily take tablets or capsule depending on their sizes. Thus, it is necessary to develop new pharmaceutical preparations that are easily taken by paediatric and patients with dsphagia. Paediatric formulations are available in different types of oral dosage forms like syrup, suspension, dry syrup, etc. But so many problems are arising in the usage of that type of formulation in paediatrics like, stability, dosage wastage, dose dumping etc. and also children's are not showing the interest to take such type of formulation.

Chewable \& disintegrating dosage forms are more convenient for administration of medication to patients with swallowing difficulties compared to liquid \& powder formulations because of difficulty in handling. Chewable formulation may require less amount of super disintegrants compared to disintegrating formulation hence it has high drug dose carrying capacity. Moreover it has pleasing taste, smooth texture, \& no unpleasant after taste. Drugs with unpleasant taste like aspirin, erythromycin, acetaminophen, ibuprofen \& antacids, minerals \& vitamin supplements can be formulated as soft chewable dosage form. Soft chewable drug delivery system will be easily accepted by children's because of its aesthetic appearance \& pleasing taste. ${ }^{1}$

\section{Jelly}

According to the17th edition of Japanese Pharmacopeia jellies meant for oral administration are non-flowable gelatinous preparations of definite size \& shape, meant for oral administration. Jellies are defined as semisolid preparations that are transparent, translucent or nongreasy, intended for internal/ external application.

Throughout years medicated jelly has gained rising acceptance as a drug delivery system. Drugs which have rapid onset of action, whose main absorption site is stomach and small intestine can be formulated as jelly. They are prepared from natural gums like tragacanth, pectin, sodium alginates or from synthetic derivatives like methyl cellulose and sodium carboxymethyl cellulose. Children \& other age group may prefer medicated jelly compare to oral liquid or tablets due its eye catching appearance, pleasant taste, \& is easy to handle. Medicated jelly can be used in local treatment of ailment related to oral cavity \& also in the treatment of systemic condition.

\section{CLASSIFICATION OF JELLIES}

Jellies can be classified as

\section{Medicated Jelly}

These are mainly used over mucous membrane and skin \& they possess spermicidal, local anaesthetics, and antiseptic properties. These jellies hold adequate amount water which after evaporation gives a local cooling effect and residual film provides protection

Example: Ephedrine sulphate jelly is used to seize the bleeding of nose since it is vasoconstrictor.

\section{Lubricating Jelly}

These jellies are intended for lubrication of equipments used in diagnosis like surgical gloves, catheters, cystoscopes

\section{Miscellaneous Jelly}

These are intended for diverse applications like electrocardiography \& patch testing

\section{Advantages}

1. It can be administer easily i.e., anywhere, anytime as it is easy to handle \& doesn't require water.

2. Therapeutic action of drug can be terminated by spitting it before complete ingestion of medicated jelly

3. It can also be used for systemic delivery of drugs, which are prone to metabolism in the gut wall or liver.

4. Moreover the drugs that are liberated \& swallowed from medicated jelly, will reach the gastrointestinal tract either in dissolved or suspended form in saliva and hence it will be easily available.

5. Delivery of therapeutic agent to systemic circulation through the oral mucosa can help to overcome the problems related to difference in drug release and retention times

6. It serves as ideal method of drug delivery for dysphasia patients as it reduces the risk of aspiration

\section{Disadvantages:}

1. As it is aqueous based preparation it needs appropriate packaging to maintain stability of drugs in various environment

2. It may lead to unpleasant taste if not formulated appropriately. ${ }^{2}$

\section{VARIOUS COMPONENTS OF MEDICATED JELLY FORMULATION}

\section{Gelling Agent}

These are usually hydrocolloids, which have been found appropriate for the formulation of gel like matrix. These gelling agents dissolve in liquid phase as colloid mixture resulting in formation of weak cohesive internal structure. Following are examples of gelling agents:

\section{Sodium Alginate}

Alginate is obtained from the cell wall of brown algae which up on binding with water forms thick gum .It is used in various oral and topical pharmaceutical formulations. It is generally used as thickening agent and suspending agent in various topical formulations such as pastes, creams and gels. It is used in cosmetics and food products as well. ${ }^{3}$

\section{Pectin}

It is a heteropolysaccharide obtained from cell walls of terrestrial plants. It is used against constipation \& diarrhoea, where it increases viscosity \& volume of stool. Due to its lesser cost it is used in various delivery methods like controlled release, mucoadhesive, gastroretentive, colon- specific drug delivery systems. Also used as stabilizer in cosmetics. ${ }^{4}$ 


\section{Tragacanth}

Tragacanth gum works as an emulsifying and suspending agent in various pharmaceutical preparations such as emulsion, gels, and creams. Also used as thickener, stabilizer, \& texturant additive in foods \& pharmaceuticals. ${ }^{5}$

\section{Gelatin}

Gelatin is generally used as gelling agent in pharmaceutical preparation, vitamin capsules, cosmetic technology, \& photographic emulsions. Also used in implantable delivery system to deliver drug suspended in biodegradable matrix. ${ }^{6}$

\section{Xanthan Gum}

It is commonly used as a thickening, emulsifying, suspending and stabilizing agents in oral, topical pharmaceutical formulations, cosmetic, and food products. Used as binder in tooth paste \& keeps the product uniform. Used as a hydrocolloid in the food preparations \& thickening agent in shampoos.?

\section{Cellulose derivatives:}

Used as emulsifier \& thickener in food \& cosmetic preparations. Also used for relief from constipation problem E.g. Methyl cellulose, Sodium carboxy methyl cellulose. $^{8}$

\section{Agar}

Agar-agar is vegetarian product \& substitute to gelatine. It is obtained from algae \& is White and semitranslucent. It has various applications such as thickener, gelling agent, texturizer, moisturizer, emulsifier, flavour enhancer, and absorbent in pharmaceuticals \& food products. $^{9}$

\section{Carrageenans}

It is obtained from extracts of red edible seaweeds, $\&$ are linear sulfated polysaccharides .They are mainly used as gelling, thickening, and stabilizing agents in food \& pharma industry. Carrageenan is vegetarian $\&$ is used as substitute for gelatine in confectionery.

There are three main types of carrageenan, which vary in their extent of sulfation. Kappa-carrageenan contain one sulfate group, iota-carrageenan have two sulphate group where as lambda-carrageenan has three sulfate group per disaccharide. ${ }^{10}$

\section{Sweetners}

\section{Sucrose}

Sucrose was most preferred sweetening agent because it is soluble in water, it is economical i.e., its highest purified form can be obtained at reasonable price, physically and chemically stable in different $\mathrm{pH}$.

It is widely used in combination with sorbitol, glycerin and other polyols to prevent crystallization of sucrose. ${ }^{11}$

\section{Dextrose}

They are anhydrous \& monohydrate form of dextrose, among them anhydrous form is hygroscopic in nature. It is made by complete hydrolysis of starch. It is roughly $70 \%$ as sweet as sucrose. ${ }^{12}$

Table 1: Various sweeteners \& its sweetness in terms of sucrose

\begin{tabular}{|l|l|}
\hline Sweetner & Sweetness corresponding to sucrose(X) \\
\hline Sucralose & $1000 \mathrm{X}$ \\
\hline Saccharin & $500 \mathrm{X}$ \\
\hline Aspartame & $250 \mathrm{X}$ \\
\hline Xylitol & $1 \mathrm{X}$ \\
\hline Dextrose & $0.75 \mathrm{X}$ \\
\hline Sorbitol & $0.5 \mathrm{X}$ \\
\hline
\end{tabular}

\section{Mannitol}

Mannitol is a white, crystalline polyol obtained by hydrogenation of fructose. It is approximately $50 \%$ as sweet as sucrose. It is freely soluble in water and it imparts a mild cooling sensation when it is chewed or dissolved in the mouth due to its negative heat of solution. Mannitol is preferred excipient in chewable tablet formulations because of smooth consistency \& other exceptional properties. It is used dusting powder on chewing gums since does not bind water well. It is thermostable \& can be used in confectionaries. ${ }^{12}$

\section{Saccharin:}

It is an artificial sweetening agent. It is about 250-500 times sweet as sucrose. Particularly at higher concentrations it gives bitter or metallic aftertaste. It has excellent stability, saccharin sodium \& calcium has excellent water solubility. ${ }^{11}$

\section{Sucralose:}

It is an artificial sweetener. Sucralose is a obtained by replacing 3 hydroxyl groups with chlorine atoms in sucrose molecule. It is about 320 to 1,000 times as sweet as sucrose, twice as sweet as saccharin, and thrice as sweet as aspartame. It does not have any calories since most of the ingested sucralose is not broken down by the body. It is thermostable and also remains stable in wide $\mathrm{pH}$ range. Hence it can be use in products that need a longer shelf life. Compared to sucrose onset of sweetness occurs slowly but sweetness remain for longer duration of time. ${ }^{11}$

\section{Sorbitol}

Sorbitol is a sugar alcohol \& isomer of mannitol. It is about $60 \%$ as sweet as sucrose.

It is obtained from corn syrup or by reduction of glucose i.e., changing the aldehyde group with hydroxyl group. It is used as humectant \& thickener in cosmetics, used as laxative, formulation of soft gel capsules \& in treatment of hyperkalaemia. It is commercially available as Sorb$\mathrm{Tab}$ and crystalline tablet type used for direct compression. ${ }^{12}$

\section{Colouring agents:}

Colourants are used for the following reasons:

1. To provide aesthetic appearance to dosage forms \& to increase patient acceptance 
2. To maintain uniformity of the preparation containing non uniformly coloured raw materials

3. Also used to match the flavour used in the formulation

4. Help in product recognition and differentiation

According to the Food drug and cosmetic Act of 1938 Colorants are classified as:

1. FD\& C colours: These are certified colorants that can be used in foods, drugs and cosmetics.

2. D\&C colours: It includes dyes and pigments that is used in drugs \& cosmetics which are meant for ingestion \& application on mucous membranes

3. External D\&C: It includes colorants that can be used in external preparations, however its use in products meant for ingestion is not considered as safe due to their oral toxicity

\section{Types of Colouring agents}

\section{Natural Colours}

It is extracted from natural sources or chemically synthesized such as beta-carotene.

\section{Mineral Colours}

Example of Mineral colour include mixture of red \& yellow ferric oxides gives flesh colour to calamine lotion

\section{Dyes}

These are synthetic chemical compounds that imparts colour when it is dissolved in a solvent such as propylene glycol and glycerine. It contains 80 to $93 \%$ pure colorant material.

\section{Lakes}

Lakes have been defined by the FDA as the aluminium salts of FD\&C water soluble dyes extended on a substratum of alumina. Lakes prepared from calcium salts of FD\&C dyes are also permitted.

\section{Flavouring Agents}

For flavour first, the inherent taste of the active drug is evaluated to determine its possible effect on the formulation, after that final decision is made depending on the influence of components on both the pharmaceutical properties and organoleptic characteristics of the formulation. ${ }^{13}$

Table 2: Flavours used as per taste

\begin{tabular}{|l|l|}
\hline Taste & Flavours used \\
\hline Acidic & Orange, lemon, cherry, grape fruit \\
\hline Alkaline & Vanilla, chocolate, mint \\
\hline Bitter & Orange, anise, lemon \\
\hline metallic & Grape, berry \\
\hline Sweet & $\begin{array}{l}\text { Honey, chocolate, raspberry, bubble gum, } \\
\text { mint }\end{array}$ \\
\hline
\end{tabular}

\section{Preservatives:}

In view of the fact that jellies are aqueous based preparations, therefore they are prone to microbial attack. Preservation is must in order to avoid at all any incompatibilities between gelling agents $\&$ to retain the shelf life of the product. Most of cellulose derivatives are used as preservatives. Following are the examples.

Methyl Paraben

Propyl Paraben

Benzoic Acid

Benzalkonium Chloride

Chlorhexidine acetate. ${ }^{14}$

\section{Stabilizers}

Generally stabilizers are incorporated with aim of maintaining desirable properties of product until it is consumed by customer. These additives that are added in jelly formulation in order to prevent the drying of jellies. Examples of stabilizers are Propylene glycol and Sorbitol.

Chelating Agents are incorporated to avoid any reactivity between base or medicament with heavy metals e.g. EDTA. ${ }^{2}$

\section{PREPARATION METHOD OF JELLY:}

1. Weighed all required ingredients accurately.

2. Sugar syrup is prepared by dissolving $67.7 \mathrm{gm}$ of sugar in a beaker containing $100 \mathrm{ml}$ water over medium flame.

3. To the following solution gelling agent will be added with continuous stirring and is heated until completely dissolved.

4. After that citric acid and stabilizer will be added with stirring to maintain $\mathrm{pH} \&$ to improve smoothness of the jelly.

5. At this moment drug is weight accurately \& dissolved in appropriate vehicle, then it is added to the above mixture with stirring.

6. Then preservative, colour, flavour will be added \& mixed thoroughly.

7. Then entire solution will be transferred into moulds and kept for cooling until it is gelled properly. Moulds are covered appropriately to avoid contact with outer environment.

\section{EVALUATION OF JELLY}

\section{Physical Examination}

The medicated jelly will be examined physically for appearance like texture, transparency and consistency, gumminess and grittiness. Grittiness is determined by rubbing the jelly between fingers

\section{2. $\mathbf{p H}$ :}

The $\mathrm{pH}$ of the jelly can be determined using digital $\mathrm{pH}$ meter. $0.5 \mathrm{gm}$ of the weighed formulation was dispersed in $50 \mathrm{ml}$ of distilled water and the $\mathrm{pH}$ was noted.

\section{Syneresis:}

Syneresis is defined as contraction \& separation of water from the gel upon storage. One of the major causes for it is using lesser concentration of gelling agent. Low acylated guar gum gels are mostly prone to syneresis

\section{Drug Content}

Ten jellies are selected \& crushed in a mortar \& then mixture equivalent to that of drug was taken \& 
dissolved in $100 \mathrm{ml}$ of volumetric flask containing 6.8 $\mathrm{pH}$ buffer \& the final volume was made up to the mark. Then the solution was filtered \& diluted appropriately, and analyzed spectrophotometrically using UV-visible double-beam spectrophotometer

\section{Dissolution}

It is performed by masticating artificially in $6.8 \mathrm{pH}$ buffer for 5 min\& then transferred in $1.2 \mathrm{pH}$ buffer. The solution was filtered \& diluted appropriately, and analyzed spectrophotometrically using UV-visible double-beam spectrophotometer.

\section{Stability Studies}

The jelly formulations are packed in aluminium foils \& stored in polyethylene containers at $0^{\circ} \mathrm{C}, 25^{\circ} \mathrm{C} / 60 \% \mathrm{RH}$ for 90 days. $^{15}$

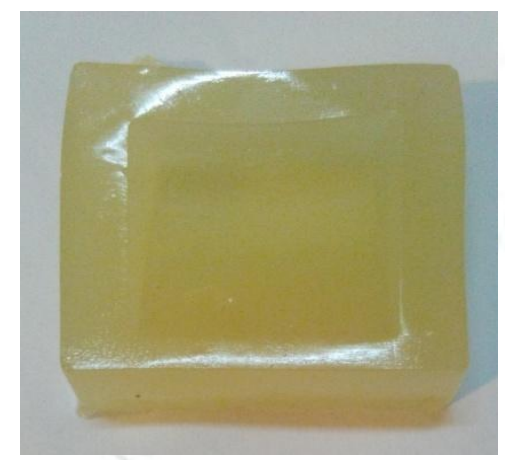

Figure 1: Jelly made using gelatine

\section{SOFT CHEWS}

Chewable formulation / soft chew are defined as solid or semi-solid dosage form containing water-soluble excipients with one or more active pharmaceutical ingredients (API) .Since it is chewable formulation it has to be chewed in between the teeth before swallowing. As a result of chewing the drug enclosed in the base is released into the saliva $\&$ there are two possible outcomes for drug absorption, firstly either it can be absorbed through the oral mucosa or might undergo GI absorption. Medicated soft chews can be used for local as well as systemic treatment of diseases since absorption through oral mucosa \& GIT occurs simultaneously.

\section{Advantages of Soft Chews}

1. Improved patient compliance, well acceptable by children

2. Drug Absorption is rapid

3. Improved bioavailability

4. Fast onset of action

5. Exhibits Local as well as systemic effect

6. Product uniqueness through marketing prospective

7. Easy to administer as it does not requires water

8. Can be used to overcome dental caries \& candidiasis

9. Treatment can be terminated at any time before swallowing
10. Can be used in dealing with xerostomia as it stimulates flow of saliva in the mouth

\section{Disadvantages of Soft Chews}

1. Prolonged chewing can results in pain in facial muscles and ear ache in children

2. Sweeteners like sorbitol can cause diarrhoea \& sucrose may cause dental caries

3. Soft chews may stick to enamel dentures and fillers.

4. Additives may possess some side effects such as cinnamon may lead to ulcers in oral cavity and liquorice may cause hypertension

5. Must kept in dry place as it is hygroscopic

6. Proper packaging is essential to keep the product safe \& stable. ${ }^{16}$

\section{Various Components in Formulation of Soft Chew}

\section{Sweetners}

\section{Sucrose}

Sucrose is a sweet disaccharide with rapid onset and least lingering .It is found in sugar cane, fruits \& has high water solubility. It breakdown into fructose and glucose which is further metabolized and can be used for energy generation. Over eating can lead to obesity. Sugar, liquid glucose \& water serves as base in soft chew. When sucrose is exposed to high temperature it becomes hard, brittle \& when treated at low temperature it becomes soft. ${ }^{17}$

Table 3: different stages of sugar

\begin{tabular}{|l|l|}
\hline Temperature & various stages of sugar \\
\hline $112^{\circ} \mathrm{C}$ & Thread stage \\
\hline $116^{\circ} \mathrm{C}$ & soft ball stage \\
\hline $120^{\circ} \mathrm{C}$ & firm ball stage \\
\hline $130^{\circ} \mathrm{C}$ & hard ball stage \\
\hline $143^{\circ} \mathrm{C}$ & soft crack stage \\
\hline $154^{\circ} \mathrm{C}$ & hard crack stage \\
\hline $170^{\circ} \mathrm{C}$ & Caramel stage \\
\hline
\end{tabular}

\section{Lactose}

Lactose is a monosaccharide obtained from whey which is a by-product obtained during production of cheese. It is $15 \%$ as sweet as sucrose hence to overcome its blandness artificial sweetener is required. ${ }^{12}$

\section{Cylamates:}

These are solid \& are sodium or potassium salts of cyclamic acid. Its use is banned in U.S. since they found it to be carcinogenic. It is $30-50$ times sweeter than sucrose. Sodium cyclamate being artificial sweetener it has less sweetness in comparison to other artificial sweeteners, so it is used in combination with sweeteners like saccharin ex: combination of 10 parts cyclamate to 1 part saccharin is mostly commonly used. It is thermostable, less costly than other sweeteners like sucralose.

\section{Aspartame:}


It is the composed of phenylalanine and aspartic acid, esterified with methanol. It is about 200 times sweeter than sucrose \& used in combination with acesulfame potassium.

\section{Liquid Glucose}

Starch up on partial hydrolysis gives concentrated aqueous solution of polysaccharides termed as liquid glucose. Based on grade it may contain different amounts of glucose, maltose and higher oligosaccharides. It mostly contains $10 \%$ to $43 \%$ glucose.

Various properties of liquid glucose are as follows:

1. Up on storing it does not crystallize

2. Used as preservative.

3. Used to provide body and cohesiveness to the products.

4. Used in combined syrups to avoid crystallization of sucrose

5. Used as stabilizer for emulsions

6. Used to impart sweetness, soften texture and volume. $^{11}$

\section{Emulsifiers}

Emulsifiers consists of hydrophilic and alipophilic parts, they are used to stabilize emulsions by reducing interfacial tension at the interface resulting in homogenous distribution of both phases in product. Emulsifiers are most commonly used additives. They are used for many reasons. It is used to maintain texture, quality $\&$ freshness of product. It is also used to inhibit growth of moulds

\section{Monoglyceride derivatives:}

Ethoxylated monoglycerides, Acetoglycerides and Diacetyl tartaric esters of monoglycerides .

\section{Fatty Acid Derivatives}

Polyglycerol esters (PGE), propylene glycol esters (PGMS), stearoyl lactylates, sucrose esters, sorbitan esters and polysorbates. Sucrose esters are also used in bubble gum.

\section{Lecithin}

Lecithin is fat based compounds present in all cells. It functions include formation of hydrophobic walls of cell membranes, very important role in brain function, transport of Vitamins such as A, D, E and K, throughout the body. It is used for health issues like multiple sclerosis, bipolar disorder and Alzheimer's disease since it is going to have effect on nerve cells. ${ }^{18}$

\section{Tween}

Polysorbate 80 used as non-ionic surfactant and emulsifier in foods and cosmetics. It is synthetic viscous yellow colour liquid \& is water-soluble. Polysorbate 80 is a derivative of polyethoxylated sorbitan and oleic acid. ${ }^{19}$

\section{Span}

Sorbitan monostearate is an ester derived from sorbitan and stearic acid. It is used as an emulsifier \& non-ionic surfactant. It consists of emulsifying, dispersing, and wetting properties. It is used in food and healthcare products.$^{20}$

\section{Fats \& Oils}

Fats and oils are important in human diet, and in foods it is used to maintain product quality. The required properties such as crystallization and melting property can not be achieved by pure fats or their mixtures, hence various modification techniques like fractionation, hardening interesterification have been utilized. Interesterification is a reaction that utilizes chemicals or enzymes as catalyst. It involves random or specific relocation of fatty acids esterified to the glycerol backbone molecule, depending on change in position, changes in some physico-chemical properties of the fat occurs. For example changes in solid fat content or crystallization behaviour.

Interesterification is a chemical reaction that leads to exchange of two fatty acids within a triacylglycerol or reorganization of fatty acids among triacyl glycerol molecules. Hence it is a two-step process consisting of

(a) Firstly hydrolysis

(b) Followed by esterification at a glycerol moiety. Various substrates along with triacylglycerols include palmitic, stearic acids, esterified fatty acids, \& alcohols. $^{21}$

\section{Condensed Milk or Milk Cream}

Condensed milk consists of cow's milk from which water is evaporated. Sweetened condensed milk is extremely thick, sweet due to added sugar which can be stored for years without refrigeration if it is not opened. Unsweetened condensed milk is evaporated milk without added sugar. ${ }^{22}$

\section{Cream}

It is the fatty layer scooped out from the top of milk before homogenization. In case of unhomogenized milk fat is less dense in nature $\&$ it rises to the top which is removed eventually. Centrifuges are used to accelerate the process of production of cream. Cream contains higher amount of saturated fat. Different grades of cream are available based on total butterfat content. $^{23}$

\section{Colour}

Colour can be defined as substance such as dye or pigment that provides colour when it is added as additive to the preparation. They are available as liquids, powders, gels, and pastes.

Seven artificial colourings that are permitted in US are as follows:

FD\&C Blue No. 1 - Brilliant Blue FCF, blue shade

$>$ FD\&C Blue No. 2 - Indigotine, indigo shade

$>$ FD\&C Green No. 3 - Fast Green FCF, turquoise shade

$>$ FD\&C Red No. 3 - Erythrosine, pink shade

$>$ FD\&C Red No. 40 - Allura Red AC, red shade

> FD\&C Yellow No. 5 - Tartrazine, yellow shade 
$>$ FD\&C Yellow No. 6 - Sunset Yellow FCF, orange shade

\section{Natural Colours}

Various natural colours are as follows:

- Carotenoids, chlorophyllin, anthocyanins, and betanin are plant pigments used to colour food products.

- Caramel colouring is obtained from caramelized sugar

- Carmine a red dye obtained from the insect Dactylopius coccus

- Others include Lycopene, Paprika, and Turmeric.

\section{Flavour}

Flavour can be defined as the sensory impression of food or any other substance. It can be determined by the chemical senses of taste and smell. Flavouring agents that can be used are essential oils and synthetic flavours. For example: citrus oils, peppermint oil, clove oil, spearmint oil, wintergreen oil, anise oil, and fruit essences. Chewable formulation of antacids, vitamins, antibiotics are flavoured sweetened to improve patient compliance. $^{24}$

Table 4: various colours used according to flavour

\begin{tabular}{|l|l|}
\hline Specific Flavour & Colour Used \\
\hline Apple, cherry & Pink-red \\
\hline Lemon & Yellow-orange \\
\hline Caramel & Brown \\
\hline
\end{tabular}

\section{Preservatives}

A preservative is can be defined as a substance that is added to products like food, pharmaceutical drugs, biological samples, cosmetics, etc to avoid decomposition by microbial growth or due to chemical changes in the product preservation is done to retain natural characteristics, appearance, to increase shelf life of product for storage .

\section{Natural Food Preservatives}

Natural food preservatives consist of salt, sugar, alcohol, vinegar. Citrus acid and ascorbic acid interfere with enzymatic metabolism leading to the preservation.

\section{Chemical Food Preservative}

They have found to be most efficient as preservative. Examples of chemical food preservatives are:

$>$ Benzoates like as sodium benzoate, benzoic acid

$>$ Nitrites like sodium nitrite

$>$ Sulphites like sulphur dioxide

$>$ Sorbates like sodium sorbate, potassium sorbate. ${ }^{25}$

\section{Sodium Benzoate}

It is the sodium salt of benzoic acid. Initially the absorption of benzoic acid into the cell occurs \& when the intracellular $\mathrm{pH}$ falls to 5 or lower, the anaerobic fermentation of glucose by phosphofructokinase thereby inhibiting the growth of microorganisms. ${ }^{26}$

\section{Methylparaben}

It is usually used in cosmetics and personal-care products. It is also an antifungal agent $\&$ food additive with E number E218. ${ }^{27}$

\section{Propylparaben}

It is n-propyl ester of p-hydroxybenzoic acid. It can be obtained from nature (plants \& some insects) \& can be produced synthetically as well. It is used in pharmaceuticals cosmetics \& foods with $\mathrm{E}$ number E216.

\section{Other includes:}

> Antioxidants: Sulfites, Vitamin E, Vitamin C and Butylated hydroxytoluene (BHT)

$>$ Chelating agent : EDTA, Polyphosphates and Citric acid. $^{28}$

\section{Preparation method of soft chew:}

1. weighed all required ingredients accurately

2. Combine sugar, water, liquid glucose $\&$ heat it over medium heat over hot plate until it is dissolved

3. Then add condensed milk/ milk cream \& heat the above over medium with stirring until it reaches $120^{\circ} \mathrm{C}$

4. Then remove the mixture from heat add vegetable fat, liquid soya lecithin \& mix thoroughly

5. Then drug \& preservative is dissolved in suitable solvent $\&$ added to the above mixture

6. Finally colour, flavour is added \& is transferred into mould, cooled at room temperature until it becomes firm enough

\section{Evaluation of soft chew}

\section{Physical Appearance}

Soft chew will be examined physically for colour odour $\&$ stickiness

\section{Weight Variation}

Twenty soft chews were selected and weighed individually. Average weight was calculated and the individual weights were compared with the average weight.

\section{Drug Content}

Ten soft chews are selected \& crushed in a mortar \& then mixture equivalent to that of drug was taken \& dissolved in $100 \mathrm{ml}$ of volumetric flask containing 6.8 $\mathrm{pH}$ buffer $\&$ the final volume was made up to the mark. Then the solution was filtered \& diluted appropriately, and analyzed spectrophotometrically using UV-visible double-beam spectrophotometer

\section{Dissolution}

It is performed by masticating artificially in $6.8 \mathrm{pH}$ buffer for 5 min\& then transferred in $1.2 \mathrm{pH}$ buffer. The solution was filtered \& diluted appropriately, and analyzed spectrophotometrically using UV-visible double-beam spectrophotometer.

\section{Stability Studies}

The soft chew formulations are packed in aluminium foils \& stored in polyethylene containers at $0^{\circ} \mathrm{C}, 25^{\circ} \mathrm{C}$ /60\% RH for 90 days. ${ }^{1}$ 

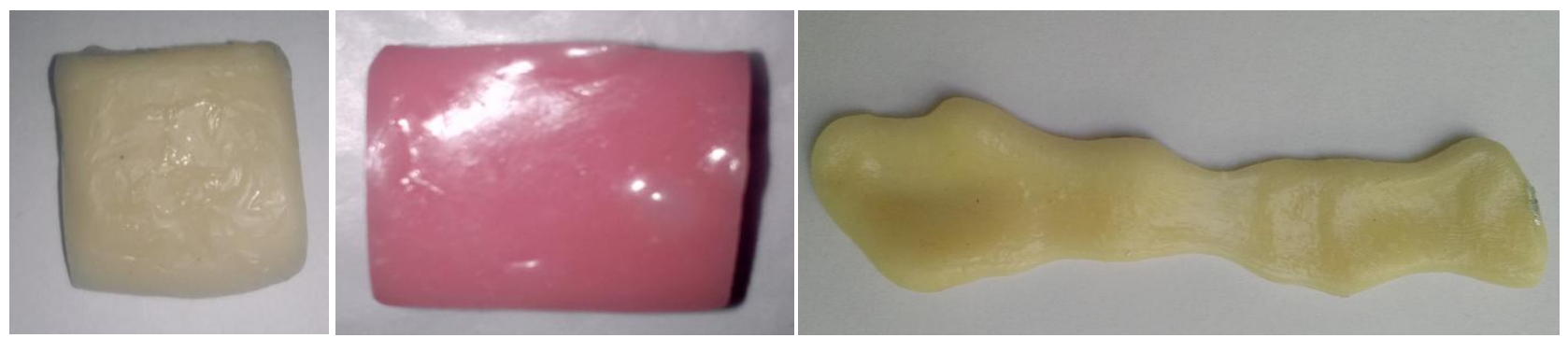

Figure 2: Soft Chew

\section{CONCLUSION}

Studies on Soft chewable oral drug delivery system for paediatrics \& dysphagia patients are going on. It has many advantages in comparison to other dosage forms

\section{REFERENCES}

1. Mishra B, Sharma G, Investigation of organoleptic characteristics in the development of soft chews of calcium carbonate as mineral supplement, Yakugaku Zasshi, 2009; 129(12):1537-1544.

2. Raja Manali M, Dhiren P, Oral medicated jelly: a recent advancement in formulation, An international journal of pharmaceutical sciences, 2016; 7(2):13-20.

3. Wikipedia contributors. Alginic acid. Wikipedia, The Free Encyclopedia. May 21, 2018, 02:49 UTC. Available at: https://en.wikipedia.org/w/index.php?title=Alginic_acid\&o ldid=842229245.

4. Wikipedia contributors. Pectin. Wikipedia, The Free Encyclopedia. June 15, 2018, 14:21 UTC. Available at: $\underline{\text { https: } / / \text { en. } \text { wikipedia.org/w/index.php?title }=\text { Pectin\&oldid }=8}$ 45988473.

5. Wikipedia contributors. Tragacanth. Wikipedia, The Free Encyclopedia. June 6, 2018, 21:26 UTC. Available at: https://en.wikipedia.org/w/index.php?title=Tragacanth\&old id $=844744223$.

6. Wikipedia contributors. Gelatin. Wikipedia, The Free Encyclopedia. June 6, 2018, 10:16 UTC. Available at: https://en.wikipedia.org/w/index.php?title=Gelatin\&oldid= 844670188.

7. Wikipedia contributors. Xanthan gum. Wikipedia, The Free Encyclopedia. June 14, 2018, 15:11 UTC. Available at: https://en.wikipedia.org/w/index.php?title=Xanthan_gum\& oldid=845848216.

8. Wikipedia contributors. Carboxymethyl cellulose. Wikipedia, The Free Encyclopedia. June 13,

2018,0:42UTC.Availableat: https://en.wikipedia.org/w/index.p hp?title=Carboxymethyl_cellulose\&oldid $=845671169$

9. Wikipedia contributors. Agar. Wikipedia, The Free Encyclopedia. June 20, 2018, 17:55 UTC. Available at: https://en. wikipedia.org/w/index.php?title=Agar\&oldid=84 6753815 .

10. Wikipedia contributors. Carrageenan. Wikipedia, The Free Encyclopedia. June 25, 2018, 20:24 UTC. Available at: https://en.wikipedia.org/w/index.php?title=Carrageenan\&ol $\mathrm{did}=847502464$.

11. Debojyoti B, organoleptic agents: adaptability, acceptability and palatability in formulations to make it lucrative. World Journal of Pharmaceutical Research .2015; (4):1573-1586.

12. Renu, Jyoti D, Chewable Tablets: A Comprehensive Review, The Pharma Innovation Journal, 2015; 4(5):100-105.

13. Eric D, Frank T, Grant E, Sweeteners: discovery, molecular design, and chemoreception, Food/Nahrung, 1991; 35(10): 1046

14. Wikipedia contributors. Preservative. Wikipedia, The Free Encyclopedia. June 12, 2018, 05:30 UTC. Available such as patient compliance, does not require water for administration, \& it remains stable. Further studies are required to know the various applications of such dosage forms.

at: https://en.wikipedia.org/w/index.php?title=Preservative\&ol did=845498444.

15. Javed H,Shah S,Formulation and Evaluation of Taste Masked Doxycycline $\mathrm{HCl}$ Medicated Jelly, Der Pharmacia Sinica, 2017; 8(2):33-39

16. Pundir S, Verma AM, Oral disintegrating preparation medicated chewing gum, Pharma Utility, 2014, Volume 8.

17. Wikipedia contributors. Candy making. Wikipedia, The Free Encyclopedia. April 30, 2018, 14:15 UTC. Available at: https://en.wikipedia.org/w/index.php?title=Candy_making \&oldid=838979532.

18. Available at:

http://www.chemistryindustry.biz/emulsifiers.html

19. Wikipedia contributors. Polysorbate 80. Wikipedia, The Free Encyclopedia. May 16, 2018, 08:37 UTC. Available at: https://en.wikipedia.org/w/index.php?title=Polysorbate 80 \&oldid $=841509853$.

20. Wikipedia contributors. Sorbitan monostearate. Wikipedia, The Free Encyclopedia. May 24, 2018, Available at: https://en.wikipedia.org/w/index.php?title=Sorbitan_monos tearate\&oldid $=842777923$.

21. Rohm H, Schaper C, Zahn S, Interesterified fats in chocolate and bakery products: A concise review,LWT - Food Science and Technology, 2018; 87:379-384.

22. Wikipedia contributors. Condensed milk. Wikipedia, The Free Encyclopedia. June 21, 2018,

17:14UTC.Availablehttps://en.wikipedia.org/w/index.php?title $=$ Condensed_milk\&oldid=846914233.

23. Wikipedia contributors. Cream. Wikipedia, The Free Encyclopedia. June 8, 2018, 03:43 UTC. Available at: https://en. wikipedia.org/w/index.php?title=Cream\&oldid=8 44924476.

24. Nanda AR, Garg KS, An update on taste masking technologies for Oral pharmaceuticals, Indian Journal Pharma. sci. 2002; 64(1).

25. Available at http://www.foodadditivesworld.com/

26. Wikipedia contributors. Sodium benzoate. Wikipedia, The Free Encyclopedia. June 13, 2018, 05:05UTC.Availableathttps://en.wikipedia.org/w/index.php?tit le=Sodium_benzoate\&oldid $=845642500$.

27. Wikipedia contributors. Methylparaben. Wikipedia, The Free Encyclopedia. June 16, 2018, 16:01 UTC. Available at: https://en.wikipedia.org/w/index.php?title=Methylparaben \&oldid $=846138187$

28. Wikipedia contributors. Propylparaben. Wikipedia, The Free Encyclopedia. March 29, 2018, 06:40UTC.Availableat: https://en.wikipedia.org/w/index.php?t $\underline{\text { itle}=\text { Propylparaben\&oldid }=833017551}$. 